\title{
Impact of bronchiectasis and trapped air on quality of life and exacerbations in cystic fibrosis
}

\author{
Leonie A. Tepper ${ }^{1,2}$, Elisabeth M.W.J. Utens ${ }^{3}$, Daan Caudri ${ }^{1}$, Aukje C. Bos ${ }^{1}$, \\ Karla Gonzalez-Graniel ${ }^{1,2}$, Hugo J. Duivenvoorden ${ }^{4}$, Els C.W. van der Wiel ${ }^{1,2}$, \\ Alexandra L. Quittner ${ }^{5}$ and Harm A.W.M. Tiddens ${ }^{1,2}$
}

Affiliations: 'Dept of Pediatric Pulmonology, Erasmus Medical Centre, Rotterdam, ${ }^{2}$ Dept of Radiology, Erasmus Medical Centre, Rotterdam, ${ }^{3}$ Dept of Child and Adolescent Psychiatry/Psychology, Erasmus Medical Centre, Rotterdam, and ${ }^{4}$ Dept of Neonatology, Erasmus Medical Centre, Rotterdam, The Netherlands. ${ }^{5}$ Depts of Psychology and Pediatrics, University of Miami, Coral Gables, FL, USA.

Correspondence: H.A.W.M. Tiddens, Molewaterplein 60, Room SP-3464, 3015 GJ Rotterdam, The Netherlands. E-mail: h.tiddensderasmusmc.nl

ABSTRACT Cystic fibrosis (CF) is primarily characterised by bronchiectasis and trapped air on chest computed tomography (CT). The revised Cystic Fibrosis Questionnaire respiratory symptoms scale (CFQ-R RSS) measures health-related quality of life.

To validate bronchiectasis, trapped air and CFQ-R RSS as outcome measures, we investigated correlations and predictive values for pulmonary exacerbations. CF patients (aged 6-20 years) underwent CT, CFQ-R RSS and 1-year follow-up. Bronchiectasis and trapped air were scored using the CF-CT scoring system. Correlation coefficients and backward multivariate modelling were used to identify predictors of pulmonary exacerbations.

40 children and 32 adolescents were included. CF-CT bronchiectasis $(\mathrm{r}=-0.38, \mathrm{p}<0.001)$ and CF-CT trapped air $(\mathrm{r}=-0.35, \mathrm{p}=0.003)$ correlated with CFQ-R RSS. Pulmonary exacerbations were associated with: bronchiectasis (rate ratio 1.10, 95\% CI 1.02-1.19; $\mathrm{p}=0.009$ ), trapped air (rate ratio 1.02 , 95\% CI 1.00 $1.05 ; \mathrm{p}=0.034$ ) and CFQ-R RSS (rate ratio $0.95,95 \%$ CI $0.91-0.98 ; \mathrm{p}=0.002$ ). The CFQ-R RSS was an independent predictor of pulmonary exacerbations (rate ratio $0.96,95 \%$ CI $0.94-0.97 ; \mathrm{p}<0.001$ ).

Bronchiectasis, trapped air and CFQ-R RSS were associated with pulmonary exacerbations. The CFQ-R RSS was an independent predictor. This study further validated bronchiectasis, trapped air and CFQ-R RSS as outcome measures in $\mathrm{CF}$.

@ERSpublications

A further validation of bronchiectasis and trapped air on CT and CFQ-R respiratory symptoms as outcome measures in CF http://ow.ly/kMGrU 


\section{Introduction}

Cystic fibrosis (CF) is a severe, life-shortening genetic disease affecting 70000 patients in the European Union and USA. The most prominent components of CF lung disease are bronchiectasis and trapped air. Bronchiectasis reflects irreversible widening of the airways and trapped air indicates small airway disease. Both bronchiectasis and trapped air typically begin in early childhood and progress slowly throughout life, eventually leading to end-stage lung disease $[1,2]$.

Bronchiectasis and trapped air are important indicators of prognosis [3-5]. Therefore, accurate and sensitive monitoring of these indicators is needed for optimal clinical management and as potential outcome measures in clinical trials. To date, forced expiratory volume in $1 \mathrm{~s}$ (FEV1), derived from pulmonary function tests (PFT), has been the central outcome measure for disease management and clinical trials. However, FEV1 is a relatively insensitive measure for detecting and monitoring disease progression [6]. Bronchiectasis, assessed with chest computed tomography (CF-CT bronchiectasis score), is more sensitive and accurate than chest radiography [6]. The literature suggests that the CF-CT bronchiectasis score is a valuable outcome measure since it is associated with pulmonary exacerbations [7, 8], is an important component of end-stage lung disease [9] and is associated with mortality [9]. It is not known whether the presence of bronchiectasis correlates with standardised, patient-reported outcome measures [10]. In adults with chronic obstructive pulmonary disease, bronchiectasis is associated with reduced health-related quality of life, as measured by the symptoms scale of the St George's Quality of Life Questionnaire [11]. We hypothesised that a similar association may exist for bronchiectasis in CF.

The importance of trapped air as an outcome measure is less well established than bronchiectasis. The volume and distribution of trapped air can be visualised well on end-expiratory chest CT [12]. Trapped air is observed in approximately two-thirds of newly diagnosed infants and it is also an important component of end-stage lung disease $[1,4,9]$. Hence, trapped air is considered a potential marker of early CF lung disease $[2-4,9]$. To date, as an outcome measure for clinical management and trials, trapped air has not yet been validated against other clinical markers of disease severity, such as pulmonary exacerbations and patient-reported respiratory symptoms on a health-related quality-of-life measure.

Standardised, well-validated measures of health-related quality of life in CF, such as the disease-specific Cystic Fibrosis Questionnaire-revised (CFQ-R) have been developed [13]. The CFQ-R consists of several domains (e.g. physical functioning, vitality, health perceptions and respiratory symptoms). The CFQ-R physical functioning scale, CFQ-R vitality scale, CFQ-R health perceptions and CFQ-R respiratory symptoms scale (CFQ-R RSS) have been shown to correlate with FEV1 [14]. The CFQ-R RSS has been utilised successfully both in controlled trials and in longitudinal studies [15-20]. The CFQ-R RSS was used as an outcome measure in several clinical trials showing responsivity to inhaled tobramycin [18], dornase alfa [19], hypertonic saline [20] and ivacaftor [16]. CFQ-R RSS was used as primary outcome in a phase III study for US Food and Drug administration (FDA) approval of aztreonam lysine for inhalation. A significant improvement in CFQ-R RSS was found in the treated versus placebo group, with continued efficacy documented in an 18-month open-label follow-up study [15, 21].

Although a minimal important difference has been established for the CFQ-R RSS, it is not clear what change in respiratory symptoms reflects the extent of bronchiectasis and trapped air [22].

The objectives of this study were to further validate bronchiectasis and trapped air as outcome measures by correlating them with CFQ-R RSS and pulmonary exacerbations. Furthermore, we aimed to validate bronchiectasis, trapped air and CFQ-R RSS by investigating their predictive value for pulmonary exacerbations in the following year.

\section{Methods}

\section{Study population}

Patients (aged 6-20 years) were diagnosed as having CF by a positive sweat test and/or genotyping for known CF mutations. We included clinically stable children and adolescents with CF, monitored at the Erasmus MC-CF Center (Rotterdam, the Netherlands), who had a CFQ-R and CT performed on the same day, at the annual check-up and at 1-year follow-up. If CT and the CFQ-R were not completed on the same day, a maximal time difference of 3 months was considered acceptable $(n=1)$. Patients in need of i.v. antibiotics for respiratory signs or symptoms at the time of the annual examination were considered unstable and excluded. This retrospective cohort study was approved by the institutional review board of the Erasmus MC-CF Center Rotterdam (MEC-2011-250).

\section{Chest CT evaluation of bronchiectasis and trapped air}

All volumetric CTs were acquired using a six-slice multi-detector CT scanner (Siemens Medical Solutions, Erlangen, Germany). Each CT consisted of a volumetric inspiratory and expiratory acquisition. 
Instructions for voluntary breath holds were given before scanning. Tube voltages of $80 \mathrm{kV}$ (patients weighing $<35 \mathrm{~kg}$ ) or $110 \mathrm{kV}$ (patients weighing $>35 \mathrm{~kg}$ ) were used with a 0.6 -s rotation time. Scanning was performed from apex to base at 1.5 pitch and $6 \times 2 \mathrm{~mm}$ collimation. Images were reconstructed with a $3.0-\mathrm{mm}$ slice thickness, $1.2-\mathrm{mm}$ increment and kernel B60s. For the inspiratory protocol a modulating current was used (Siemens) with a reference tube current-time product of $20 \mathrm{~mA}$, for optimal image quality. For expiratory CTs a tube current fixed at $25 \mathrm{~mA}$ with an effective tube current-time product of $10 \mathrm{~mA}$ (the typical value for a 5-year-old child) was used. This produced a lower radiation dose than the inspiratory protocol with sufficient image quality. Total radiation dose was in the order of $1 \mathrm{mSv}$.

All CTs were scored using the CF-CT scoring system, a modified version of Brody II scoring, evaluating the five lung lobes and the lingula as a sixth lobe for severity, extent of central and peripheral bronchiectasis, airway wall thickening, central and peripheral mucus plugging, opacities (atelectasis, consolidation and ground-glass pattern) and cysts and bullae on inspiratory CTs and the pattern and extent of trapped air on expiratory CTs $[7,23]$. The maximum possible composite CT score is 207 points. For statistical analysis, composite and component CT scores were expressed as a percentage of the maximum possible score $(0-$ 100). All scans were de-identified, using Myrian (Intrasense, Montpellier, France), and scored in random order by an observer blinded to clinical background [7]. To test intraobserver agreement, observer one rescored 25 random scans after 1 month. A second observer scored 25 random scans to assure a good interobserver agreement. Both observers were trained in CF-CT scoring and began scoring the study CTs after establishing good intra- and interobserver agreement.

\section{CFQ-R}

Three age-appropriate versions of the Dutch CFQ-R were administered, using a multi-informant approach (table 1): 1) the CFQ-R Child version (ages 6-13 years; 35 items covering eight domains); 2) CFQ-R Parent version (caregivers of children aged 6-13 years; 43 items covering 11 domains); and 3) CFQ-R Teen/Adult version (ages $\geqslant 14$ years; 47 items covering 12 domains) [13]. In addition to analysing the CFQ-R RSS, we analysed three health-related secondary domains: CFQ-R physical functioning, CFQ-R vitality and CFQ-R health perceptions scales. Unfortunately, the CFQ-R vitality and CFQ-R health perceptions do not exist for younger children (CFQ-R Child version).

All scale scores were standardised on a 0-100 scale, with higher scores indicating better health-related quality of life [13].

\section{PFT and pulmonary exacerbations}

PFT results (diagnostic system: Jaeger AG, Würzburg, Germany) were expressed as percentages of predictive values, according to STANOJEVIC et al. [24] for forced vital capacity (FVC) and FEV1 and ZAPLETAL et al. [25] for forced expiratory flow at 75\% of FVC.

Because there is no consensus on the definition of pulmonary exacerbations, they were conservatively defined as episodes of treatment with i.v. antibiotics for pulmonary indications in the year following administration of CT and CFQ-R [7, 8]. Pseudomonas aeruginosa positivity was defined as the presence of at least one and less than three positive respiratory cultures in the year previous to the CT scan. Chronic colonisation with Pseudomonas aeruginosa was defined as three or more consecutive positive respiratory cultures.

TABLE 1 Selected Cystic Fibrosis Questionnaire-revised (CFQ-R) scores for children, their parents and adolescents

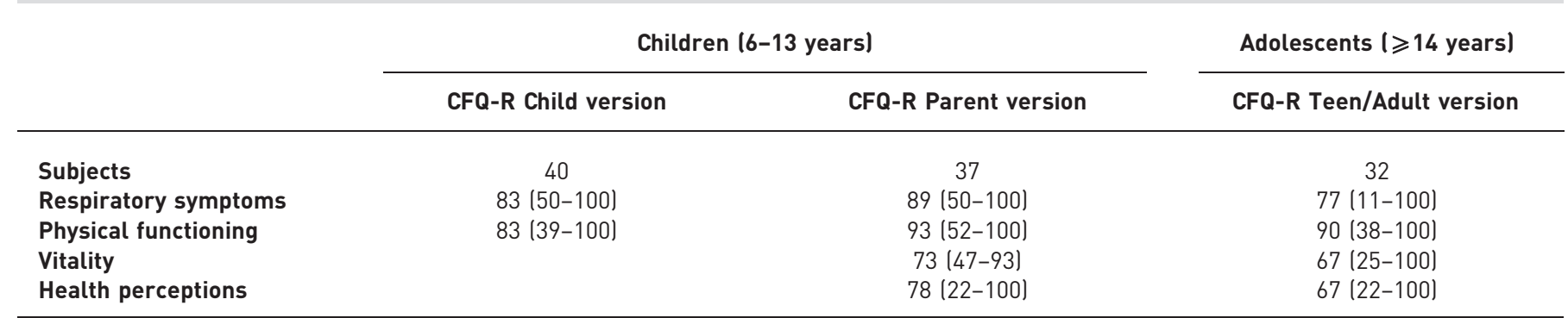

Data are presented as $n$ or median (range). CFQ-R scale scores are shown for the different versions of the CFQ-R. The CFQ-R respiratory symptoms scale was the primary target. Physical functioning, vitality and health perceptions were secondary domains correlating with forced expiratory volume in $1 \mathrm{~s}$. For each domain, a score of $0-100$ is calculated. Higher scores indicate better health-related quality of life. 


\section{Statistical analysis}

Inter- and intraobserver agreement of CF-CT scores were calculated using intra-class correlation coefficients (ICC) $(0.40-0.60$ moderate; $0.60-0.80$ good; and $\geqslant 0.80$ very good agreement). In case of low or moderate agreement between the observers, Bland-Altman plots were calculated and used to visualise whether one over- or underestimated the CT scores on the different indices [26]. Spearman's correlation coefficients were used to correlate CF-CT bronchiectasis and CF-CT trapped air scores with CFQ-R RSS, CFQ-R physical functioning, CFQ-R vitality and CFQ-R health perceptions scale scores. Negative binomial regression models were used to investigate the association between CF-CT bronchiectasis, CF-CT trapped air and CFQ-R RSS and the number of pulmonary exacerbations in the subsequent year. A multivariate model was evaluated (backward, stepwise approach) to identify independent predictors of pulmonary exacerbations in the subsequent year. In order to reach sufficient power the univariate and multivariate regression analyses were performed on the complete study population $(n=72)$. Analyses were repeated using the CFQ-R Child version and CFQ-R Parent version in children aged 6-13 years. In our final model, the CFQ-R Child version was used, because it is better to use the patient's own report on his/her symptoms as recommended by the FDA [27] and the European Medicines Agency. To interpret our results in clinical terms, we used a logistic model.

Statistical analyses were performed using SAS version 9.2 (SAS Institute Inc., Cary, NC, USA). Results are displayed as median (range) unless otherwise defined. Two-tailed testing was performed. $\mathrm{p}$-values $<0.05$ were considered to be significant.

\section{Results}

Study population

72 patients (40 children and 32 adolescents) had 72 CTs and PFTs completed. Baseline characteristics are shown in table 2. A total of 109 CFQ-Rs were collected: 40 CFQ-R Child version; 37 CFQ-R Parent version; and 32 CFQ-R Teen/Adult version. Three parents did not return the CFQ-R (see table 1). ICCs for intraobserver agreement ranged from 0.68 (CF-CT trapped air score) to 0.98 (CF-CT bronchiectasis score), whereas interobserver agreement ranged from 0.50 (CF-CT trapped air score) to 0.91 (CF-CT total score).

\section{Correlations between $C T$ and $C F Q-R$}

In children the CF-CT airway wall thickening $(\mathrm{p}<0.001)$, mucus plugging $(\mathrm{p}<0.001)$ and opacities $(\mathrm{p}=0.007)$ were significantly correlated with the CFQ-R RSS (table 3$)$. Similarly, CF-CT airway wall thickening $(p=0.01)$ and mucus plugging $(p=0.008)$ were significantly correlated with the CFQ-R RSS scores in the parent questionnaires, but, in addition, so was the CF-CT bronchiectasis score $(\mathrm{p}=0.033)$. Similar associations were found among adolescents: CF-CT bronchiectasis score $(p=0.007)$, airway wall thickening $(\mathrm{p}=0.005)$, mucus plugging $(\mathrm{p}=0.004)$ and opacities $(\mathrm{p}=0.004)$ all significantly correlated with the CFQ-R RSS scores.

\section{TABLE 2 Baseline characteristics of the study cohort}

\begin{tabular}{|c|c|c|c|}
\hline & Total & Children & Adolescents \\
\hline Subjects & 72 & 40 & 32 \\
\hline Male & $35(48.6)$ & $20(50)$ & $15(46.9)$ \\
\hline Age years & $13.4(6-20)$ & $11.5(6-14)$ & $16.5(14-20)$ \\
\hline FEV $1 \%$ predicted & $83.4(22-110)$ & $85.7(31-110)$ & $75.9(22-110)$ \\
\hline FVC $\%$ predicted & $91.9(32-119)$ & $97.9(53-119)$ & $85.9(32-112)$ \\
\hline FEF75 \% predicted & $48.5(6-95)$ & $48(7-95)$ & $49.2(6-92)$ \\
\hline Positive Pseudomonas aeruginosa culture ${ }^{\#}$ & $26(36)$ & $10(25)$ & $16(50)$ \\
\hline Chronic infection with Pseudomonas aeruginosa & $19(26)$ & $5(13)$ & $14(44)$ \\
\hline CF-CT total score \% & $7.8(0-33)$ & $5.2(0-20)$ & $11.8(0-33)$ \\
\hline Bronchiectasis score \% & $0.0(0-26)$ & $0(0-19)$ & $2.6(0-26)$ \\
\hline Airway wall thickening score $\%$ & $8.3(0-37)$ & $4.9(0-33)$ & $14.8(0-37)$ \\
\hline Mucus plugging score $\%$ & $8.3(0-50)$ & $5.6(0-50)$ & $16.7(0-42)$ \\
\hline Opacities \% & $5.6(0-19)$ & $4.6(0-13)$ & $7.4(0-19)$ \\
\hline Trapped air \% & $36.7(0-97)$ & $33.3(0-70)$ & $43.3(7-97)$ \\
\hline
\end{tabular}

Data are presented as $\mathrm{n}, \mathrm{n}(\%)$ or median (range). FEV1: forced expiratory volume in $1 \mathrm{~s}$; FVC: forced vital capacity; FEF75: forced expiratory flow at $75 \%$ of FVC; CF-CT: cystic fibrosis computed tomography scoring system. ${ }^{*}$ : includes all positive Pseudomonas aeruginosa cultures positive in the year prior to computed tomography and Cystic Fibrosis Questionnaire-revised; " chronic infection with P. aeruginosa defined as three or more consecutive positive respiratory cultures for $P$. aeruginosa. 


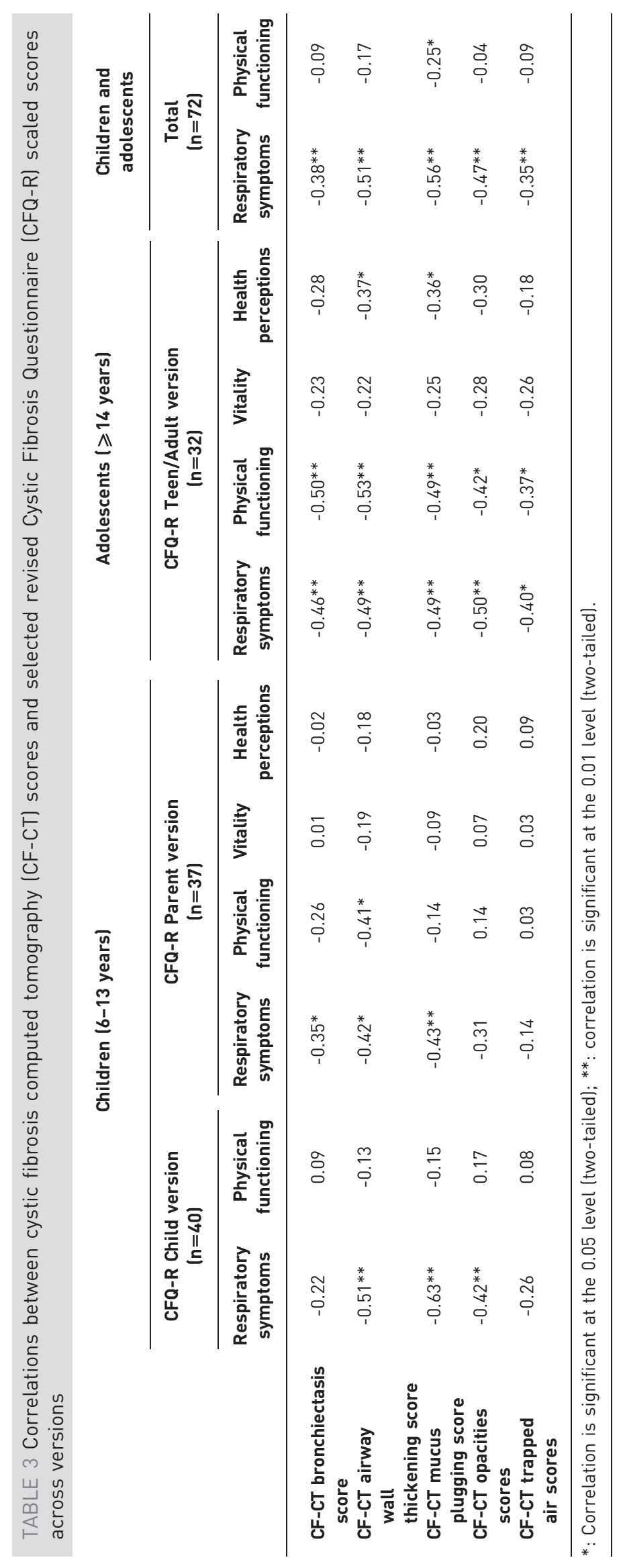




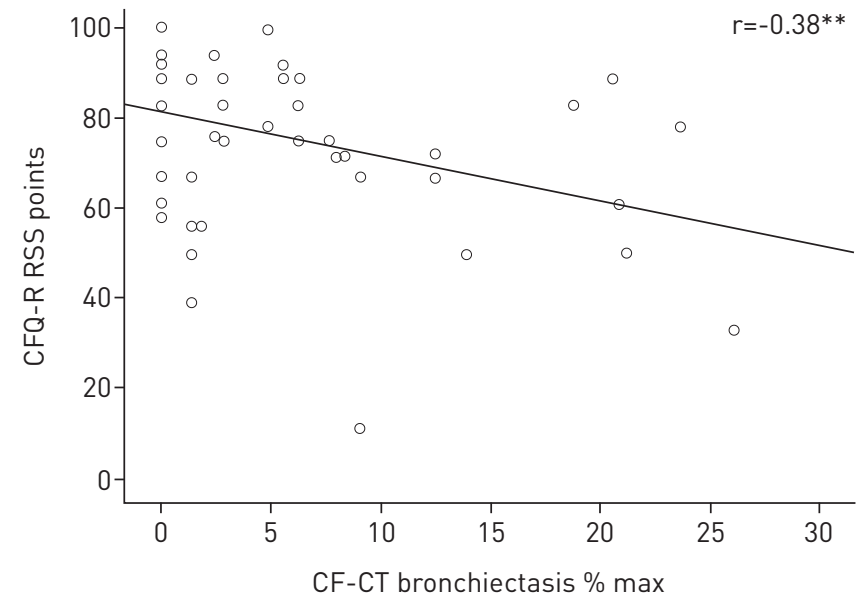

FIGURE 1 Correlation between Cystic Fibrosis Questionnaire-revised respiratory symptoms scale (CFQ-R RSS) and cystic fibrosis computed tomography (CF-CT) bronchiectasis score across ages. $\mathrm{n}=72$. ${ }^{* *}: \mathrm{p}<0.01$.

No significant correlations were found between the CF-CT scores and the physical functioning scale in children, whereas in adolescents all of the CF-CT scores correlated.

In the other health-related secondary domains (vitality and health perceptions), only CFQ-R health perceptions in adolescents was significantly associated with CF-CT airway wall thickening $(\mathrm{p}=0.038)$ and CF-CT mucus plugging $(\mathrm{p}=0.041)$.

Across ages $(n=72)$, CF-CT bronchiectasis scores were significantly correlated with CFQ-R RSS $(r=-0.38$, $\mathrm{p}<0.001$ ), with more structural changes being associated with worse respiratory symptoms (fig. 1). This relationship was also present between CF-CT trapped air scores and CFQ-R RSS ( $r=-0.35, p=0.003$ ) (fig. 2).

Associations between CT, CFQ-R RSS and pulmonary exacerbations in the following year

Associations between CT, CFQ-R RSS and pulmonary exacerbations in the year following CT and CFQ-R RSS are shown in table 4.

CF-CT bronchiectasis scores were significantly associated with the number of pulmonary exacerbations in the following year (rate ratio 1.10, 95\% CI 1.02-1.19; $\mathrm{p}=0.009$ ). This indicates that the expected number of pulmonary exacerbations increased by $10 \%$ in the following year (95\% CI 2.4-19\%) for each point increase in a patient's CF-CT bronchiectasis score. The rate ratio for CF-CT trapped air on pulmonary exacerbations in the following year was smaller, but also significant (rate ratio 1.02, 95\% CI 1.00-1.05; $\mathrm{p}=0.034$ ). CFQ-R RSS were associated with pulmonary exacerbations in the following year (rate ratio $0.95,95 \%$ CI $0.91-0.98$; $\mathrm{p}=0.002$ ). Thus, the expected number of pulmonary exacerbations decreased $5 \%$ for each one-point increase in CFQ-R RSS scores.

\section{Prediction model for pulmonary exacerbations}

72 patients had complete longitudinal data for the multivariate prediction model. Due to the relatively small sample size, a limited number of predictors were tested: sex, age, CF-CT bronchiectasis score, CF-CT trapped air score, CFQ-R RSS and positive culture for P. aeruginosa in the year before the CT. In the final

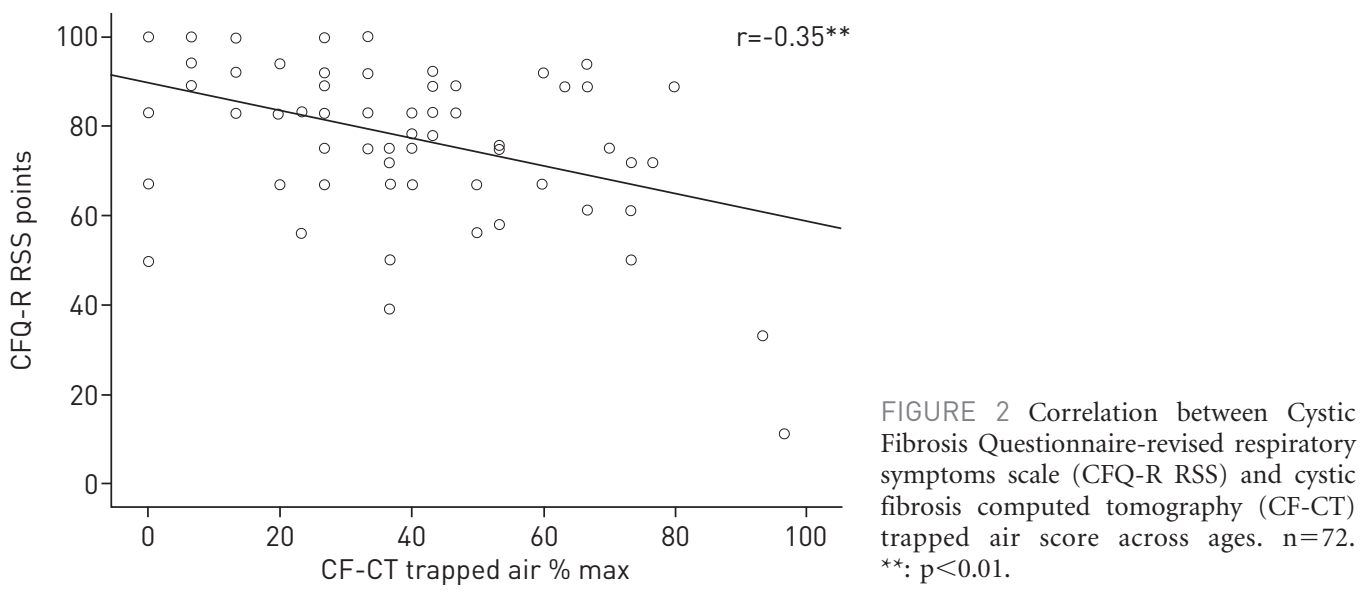


TABLE 4 Univariate and multivariate associations with pulmonary exacerbations in the year following computed tomography and completion of the Cystic Fibrosis Questionnaire-revised (CFQ-R)

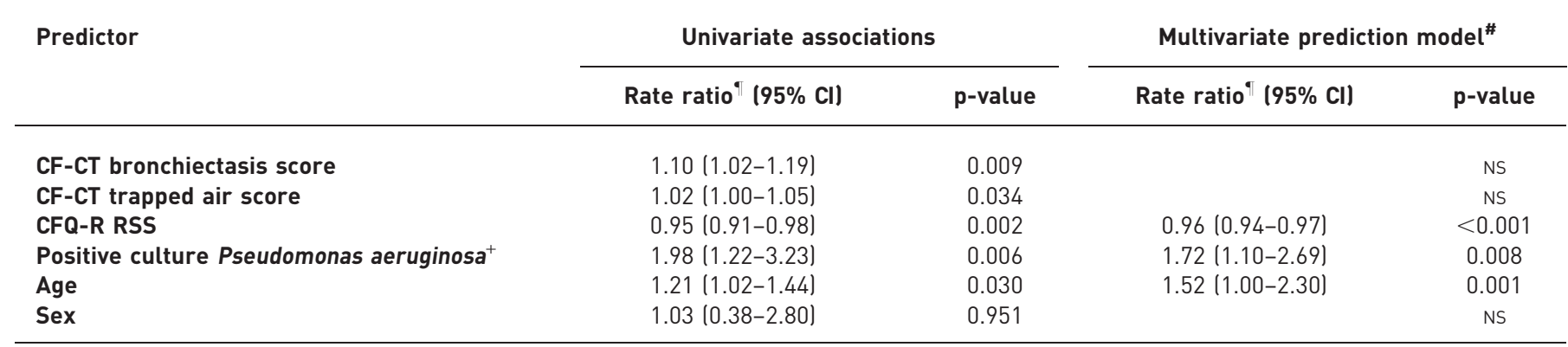

Data are presented as rate ratio (95\% CI), unless otherwise stated. $n=72$. In young children (6-13 years) the CFQ-R Child version was used. CF-CT: cystic fibrosis computed tomography; CFQ-R RSS: revised Cystic Fibrosis Questionnaire respiratory symptoms scale; NS: not significant. \#: only variables significant at the $p=0.05$ level are included in the multivariable model; " : rate ratio for scores calculated per point increase in score; ${ }^{+}$: defined as at least one positive respiratory culture for $P$. aeruginosa in the year before computed tomography and completion of CFQ-R.

model, significant predictors of subsequent pulmonary exacerbations were: age $(p=0.001)$, CFQ-R RSS $(\mathrm{p}<0.001)$, and positive cultures for P. aeruginosa $(\mathrm{p}=0.008)$ (table 4$)$. A decrease of one point in CFQ-R RSS scores predicted a $4.7 \%$ (95\% CI 3.0-6.3\%) increase in pulmonary exacerbations in the following year. QUITTNER et al. [22] showed that a four-point reduction in CFQ-R RSS scores was clinically meaningful. According to the logistic model used in our study, this would equate to a $20 \%\left(4.7^{4}\right)$ increase in the number of pulmonary exacerbations in the following year. To determine whether CFQ-R scores were merely a reflection of the number of pulmonary exacerbations in the previous year, we performed a sensitivity analysis adding the number of pulmonary exacerbations in the previous year to the multivariate model. Interestingly, CFQ-R RSS scores continued to add predictive value for number of pulmonary exacerbations in the following year $(2.6 \%$ decrease per point in CFQ-R, 95\% CI $0.4-4.8 \% ; \mathrm{p}=0.014)$. CF-CT bronchiectasis and trapped air did not remain significant in the multivariate model. No meaningful differences were present when the analysis was performed using the CFQ-R Parent version in place of the CFQ-R Child version.

\section{Discussion}

This is the first study to investigate the relationship between bronchiectasis and trapped air, assessed by chest CT and CFQ-R RSS scores. The most important finding was that more severe bronchiectasis was significantly associated with worsening respiratory symptoms. Bronchiectasis, trapped air and CFQ-R RSS were all significantly associated with pulmonary exacerbations in the following year.

The importance of bronchiectasis in CF lung disease has been well established [1, 3-5, 7-9]; however, the impact of bronchiectasis on patient-reported outcome measures in CF had not previously been examined [10]. Our finding that bronchiectasis and CFQ-R RSS are negatively associated supports the validity of bronchiectasis as a clinically relevant outcome measure. Previous studies have shown that bronchiectasis was associated with pulmonary exacerbations in the following 2 years $[7,8]$, while our data showed a similar association over a 1-year time period. Unfortunately, in our multivariate model, CF-CT bronchiectasis did not remain a significant predictor for pulmonary exacerbations, probably because we have a patient population with very mild CF, reflected by a median bronchiectasis score of 0.0 (table 2).

Although trapped air has been less well validated as an outcome measure, we found a significant, independent correlation between trapped air and CFQ-R RSS. Although the associations between CF-CT trapped air scores and both CFQ-R RSS and pulmonary exacerbations were significant, they were not as strong as the associations with bronchiectasis. This may be because trapped air has less impact on patient functioning than bronchiectasis and can be reversible to some extent $[1,2]$. A recent study showed that patients with severe advanced lung disease, such as bronchiectasis, had a higher mortality risk compared to patients who predominantly had trapped air [9]. Our results suggested that trapped air can also be considered as a valuable CT-related surrogate outcome measure in CF.

Interestingly, we also found significant associations in other CT indices: CF-CT airway wall thickening, mucus plugging and opacities were significantly associated with the CFQ-R RSS in children and adolescents. Airway wall thickening and mucus plugging are considered to be early indicators of developing disease, in contrast to bronchiectasis, which is considered to be advanced structural damage in the larger airways. Therefore, it is not surprising that these early indicators, and not bronchiectasis, were significantly 
associated with the CFQ-R RSS in the younger age group. Note that this was a young population with very mild CF lung disease. This further validates the CFQ-R RSS as a sensitive measure of structural lung damage in the early stages of CF lung disease.

We found that the lower the CFQ-R RSS score, the higher the risk for pulmonary exacerbations, irrespective of other predictors. This innovative result is consistent with the research of BRITTO et al. [28]. They concluded that pulmonary exacerbations have a profound negative impact on health-related quality of life in CF children, using a generic instrument (Child Health Questionnaire). Furthermore, our data suggested that the CFQ-R RSS is sensitive to early minor respiratory symptoms preceding pulmonary exacerbations. Therefore the CFQ-R RSS may allow earlier detection of disease progression.

In our multivariate model, after including CFQ-R RSS, CT had no added value to predict the frequency of pulmonary exacerbations. It is important to realise that the CFQ-RSS and CT provide different information. The CFQ-R RSS focuses exclusively on the frequency and severity of respiratory symptoms, whereas CT provides critical information about structural lung changes such as the extent of bronchiectasis and trapped air. CT also provides information about airway wall thickening and mucus plugging, which can be considered early leading indicators of developing disease. Consequently, aggressive treatment to prevent structural lung damage should be considered if either indicator is present on CT. Therefore, both CT and CFQ-R RSS are considered important outcome measures.

\section{Limitations}

This was a retrospective study. Therefore, we selected a robust, conservative definition of pulmonary exacerbations that were unlikely to be missed [7, 8, 29, 30]. Data were collected from a single centre, which may reduce the generalisability of the results. Furthermore, we used an age-range of 6-20 years; whether similar correlations exist in infant or adult CF populations requires further study. Considering the progressive nature of $\mathrm{CF}$, we would expect a higher rate of pulmonary exacerbations in adults and, thus, stronger associations.

In conclusion, we showed that in children with $\mathrm{CF}$, more severe bronchiectasis and trapped air were associated with worse CFQ-R RSS scores. Bronchiectasis, trapped air and the CFQ-R RSS were significantly associated with pulmonary exacerbations in the following year. Our findings validate the importance of CT measures of bronchiectasis and trapped air and the CFQ-R RSS as clinically relevant outcome measures for CF.

\section{References}

1 Mott LS, Park J, Murray CP, et al. Progression of early structural lung disease in young children with cystic fibrosis assessed using CT. Thorax 2012; 67: 509-516.

2 Hall GL, Logie KM, Parsons F, et al. Air trapping on chest CT is associated with worse ventilation distribution in infants with cystic fibrosis diagnosed following newborn screening. PLoS One 2011; 6: e23932.

3 Stick SM, Brennan S, Murray C, et al. Bronchiectasis in infants and preschool children diagnosed with cystic fibrosis after newborn screening. J Pediatr 2009; 155: 623-628.

4 Sly PD, Brennan S, Gangell C, et al. Lung disease at diagnosis in infants with cystic fibrosis detected by newborn screening. Am J Respir Crit Care Med 2009; 180: 146-152.

5 Pillarisetti N, Linnane B, Ranganathan S, et al. Early bronchiectasis in cystic fibrosis detected by surveillance CT. Respirology 2010; 15: 1009-1011.

6 de Jong PA, Lindblad A, Rubin L, et al. Progression of lung disease on computed tomography and pulmonary function tests in children and adults with cystic fibrosis. Thorax 2006; 61: 80-85.

7 Loeve M, Gerbrandts K, Hop WC, et al. Bronchiectasis and pulmonary exacerbations in children and young adults with cystic fibrosis. Chest 2011; 140: 178-185.

8 Brody AS, Sucharew H, Campbell JD, et al. Computed tomography correlates with pulmonary exacerbations in children with cystic fibrosis. Am J Respir Crit Care Med 2005; 172: 1128-1132.

9 Loeve M, van Hal PT, Robinson P, et al. The spectrum of structural abnormalities on CT scans from patients with CF with severe advanced lung disease. Thorax 2009; 64: 876-882.

10 Quittner AL, Alpern AN, Kimberg CI. Integrating patient-reported outcomes into research and clinical practice. In: Wilmott RW, Boat TF, Bush A, et al. Kending and Chernick's Disorders of the Respiratory Tract in Children. 8th Edn. Philadelphia, Saunders, 2012; pp. 251-260.

11 Martínez García MA, Perpiñá Tordera M, Román Sánchez P, et al. Consistencia interna y validez de la versión española del St. George's Respiratory Questionnaire para su uso en pacientes afectados de bronquiectasias clínicamente estables [Internal consistency and validity of the Spanish version of the St George's Respiratory Questionnaire for use in patients with clinically stable bronchiectasis]. Arch Bronchoneumol 2005; 41: 110-117.

12 Bonnel AS, Song SM, Kesavarju K, et al. Quantitative air-trapping analysis in children with mild cystic fibrosis lung disease. Pediatr Pulmonol 2004; 38: 396-405.

13 Quittner AL, Sawicki GS, McMullen A, et al. Erratum to: Psychometric evaluation of the Cystic Fibrosis Questionnaire-Revised in a national, US sample. Qual Life Res 2012; 21: 1279-1290.

14 Quittner AL, Schechter MS, Rasouliyan L, et al. Impact of socioeconomic status, race, and ethnicity on quality of life in patients with cystic fibrosis in the United States. Chest 2010; 137: 642-650.

15 Retsch-Bogart GZ, Quittner AL, Gibson RL, et al. Efficacy and safety of inhaled aztreonam lysine for airway pseudomonas in cystic fibrosis. Chest 2009; 135: 1223-1232. 
Ramsey BW, Davies J, McElvaney NG, et al. A CFTR potentiator in patients with cystic fibrosis and the G551D mutation. N Engl J Med 2011; 365: 1663-1672.

17 Sawicki GS, Rasouliyan L, McMullen AH, et al. Longitudinal assessment of health-related quality of life in an observational cohort of patients with cystic fibrosis. Pediatr Pulmonol 2011; 46: 36-44.

18 Quittner AL, Buu A. Effects of tobramycin solution for inhalation on global ratings of quality of life in patients with cystic fibrosis and Pseudomonas aeruginosa infection. Pediatr Pulmonol 2002; 33: 269-276.

19 Johnson JA, Connolly M, Zuberbuhler P, et al. Health-related quality of life for adults with cystic fibrosis: a regression approach to assessing the impact of recombinant human DNase. Pharmacotherapy 2000; 20: 1167-1174.

20 Donaldson SH, Bennett WD, Zeman KL, et al. Mucus clearance and lung function in cystic fibrosis with hypertonic saline. N Engl J Med 2006; 354: 241-250.

21 Oermann CM, Retsch-Bogart GZ, Quittner AL, et al. An 18-month study of the safety and efficacy of repeated courses of inhaled aztreonam lysine in cystic fibrosis. Pediatr Pulmonol 2010; 45: 1121-1134.

22 Quittner AL, Modi AC, Wainwright C, et al. Determination of the minimal clinically important difference scores for the Cystic Fibrosis Questionnaire-Revised respiratory symptom scale in two populations of patients with cystic fibrosis and chronic Pseudomonas aeruginosa airway infection. Chest 2009; 135: 1610-1618.

23 Brody AS, Kosorok MR, Li Z, et al. Reproducibility of a scoring system for computed tomography scanning in cystic fibrosis. J Thorac Imaging 2006; 21: 14-21.

24 Stanojevic S, Wade A, Stocks J, et al. Reference ranges for spirometry across all ages: a new approach. Am J Respir Crit Care Med 2008; 177: 253-260.

25 Zapletal A, Naidr J, Pohunek P. Strucny popis metod vysetreni funkce plic v detskem a mladistvem veku [A brief description of methods for studying pulmonary function in children and adolescents]. Cesk Pediatr 1992; 47: 520-523.

26 Bland JM, Altman DG. Statistical methods for assessing agreement between two methods of clinical measurement. Lancet 1986; 1: 307-310.

27 US Food and Drug Administration. Guidance for industry patient-reported outcome measures: use in medical product development to support labeling claims. Center for Biologics Evaluation and Research, US Dept of Health and Human Services, 2009.

28 Britto MT, Kotagal UR, Hornung RW, et al. Impact of recent pulmonary exacerbations on quality of life in patients with cystic fibrosis. Chest 2002; 121: 64-72.

29 Ortiz JR, Neuzil KM, Victor JC, et al. Influenza-associated cystic fibrosis pulmonary exacerbations. Chest 2010; 137: $852-860$.

30 Sanders DB, Hoffman LR, Emerson J, et al. Return of FEV1 after pulmonary exacerbation in children with cystic fibrosis. Pediatr Pulmonol 2010; 45: 127-134. 